\title{
Measurement of Pelvic Tilting Angle During Total Hip Arthroplasty Using a Computer Navigation System
}

\author{
S.Nishihara ${ }^{1}$, N.Sugano ${ }^{2}$, K.Nakahodo $^{3}$, T.Sasama $^{3}$, T.Nishii ${ }^{2}$, Y.Sato $^{3}$, \\ S.Tamura $^{3}$, K.Yonenobu ${ }^{2}$, H.Yoshikawa ${ }^{2}$, and T.Ochi ${ }^{1}$ \\ ${ }^{1}$ Department of Computer Aided Orthopaedic Surgery, Osaka University Medical School, \\ 2-2 Yamadaoka, Suita 565-0871, Osaka, Japan \\ ${ }^{2}$ Department of Orthopaedic Surgery, Osaka University Medical School, \\ 2-2 Yamadaoka, Suita 565-0871, Osaka, Japan \\ ${ }^{3}$ Division of Functional Diagnostic Imaging, Osaka University Medical School, \\ 2-2 Yamadaoka, Suita 565-0871, Osaka, Japan \\ Correspondence should be sent to S.Nisihihara, MD. \\ FAX: +81-6-6879-3559, email: nishihara@caos.med.osaka-u.ac.jp
}

\begin{abstract}
The purpose of this study was to measure pelvic orientation during total hip arthroplasty ( THA ) in a lateral decubitus position using a computer navigation system with an optical localizer ( OPTOTRAK ). THA was performed in 17 hips. Much attention was paid to set the patients in neutral axial rotation with the anatomical plane of the pelvis perpendicular to the operating table. After shape-based registration, pelvic orientation was tracked with light emitting diode markers fixed to the pelvis. Measurements were based on the anatomical plane. Mean movement of the pelvis from the supine position to the dislocated lateral decubitus position was 8 degrees posterior ( 26 posterior to 6 anterior), 3 degrees abducted (6 adduction to 20 abduction), and 4 degrees internally rotated ( 24 internal to 5 external). This study showed that the pelvis was not always placed in neutral axial rotation, despite the surgical plan. It was further tilted posteriorly and rotated internally when the socket was inserted. This tilt can lead to decreased socket anteversion with conventional alignment guide systems, while unknown pelvic orientation in general can cause socket malposition with such systems. Therefore, intraoperative three-dimensional measurements of pelvic orientation seems to be useful to avoid socket malposition.
\end{abstract}

\section{Introduction}

Socket malposition has been reported to be one of the causes of dislocation, impingement, wear, and loosening after total hip arthroplasty ( THA $)^{3), 4), 7)}$. Socket anteversion is affected by the flexion and rotational angles of the pelvis ${ }^{1)}$. Therefore, it is important for surgeons to know the three-dimensional orientation of the pelvis on the operating table, especially in the lateral decubitus position ${ }^{2), 55), 8)}$. The position of the pelvis may change during various operative maneuvers, even if surgeons initially pay sufficient attention to neutrally align the pelvis on the operating table. The purpose of this study is to measure the orientation of the pelvis on the operating table 
during THA procedures using a computer navigation system with an optical sensor ( OPTOTRAK ), and to estimate the risk of socket malposition with conventional alignment guides.

\section{Materials and Methods}

Our navigation system consists of the following three steps: (1) making a computer model from preoperative CT data of the object to be used in the operation; (2) registration of the computer model to the real object; and (3) tracking and measurement of the object and operative tools during operation. Preoperatively, transverse images from the level of the superior anterior iliac spines to the level of the femoral canal isthmus are obtained using a helical CT scanner. 3D acetabular bone surface models are reconstructed from this CT data. A pelvic coordinate system is defined using the inter-teardrop line and the standard anatomic pelvic plane through the superior anterior iliac spines and the pubic tubercles. THA was performed in 17 hips with osteoarthritis using this computer navigation system. Mean age at surgery was 54 years ( range, 45-68 years ).

In the operating room, the optical three-dimensional position sensor (OPTOTRAK 3020, Northern Digital Inc., Waterloo, Canada) was placed at the wall caudal to the patient. The operating table was placed so that the surgical area was within 2.5 meters from the position sensor. Patients were placed in a lateral decubitus position on the operating table such that the anatomical plane of the pelvis was perpendicular to the plane of the operating table. A posterolateral approach was used. A flat plate with 6 LED markers was fixed with a rod to the iliac crest using an extraskeletal fixation system ( Hoffmann system, Howmedica, Rutherford, USA ).

Shape-based surface registration of the previously constructed bone models to the real objects was performed in two steps by using the ICP algorithm with the least square method. First, the surgeon digitized four surface points to provide the starting position for matching, and then 30 surface points were digitized for the final registration. After shape-based surface registration, LED markers fixed to the pelvis and femur allowed for continuous intraoperative tracking of the position and movement of these bones. Orientation of the pelvis was determined based on the anatomical plane. The pelvic orientation, as measured by flexion, abduction and axial rotation, was measured in the supine and lateral decubitus positions, as well as a dislocated position when the socket was inserted.

\section{Results}

The mean pelvic tilting angle in the supine position was 6 degrees anterior ( standard deviation ( SD ): 5.8 , range: 4 posterior to 16 anterior ). The mean abduction angle in the supine position was 0 degrees ( $\mathrm{SD}$ : 4.5 , range: 14 adduction to 9 abduction). The mean axial rotation angle in the supine position was 1 degree ( SD: 2.9 , range: 6 internal to 4 external ).

Mean movement of the pelvis from the supine position to the initial lateral decubitus position was 3 degrees anterior ( SD: 7.1, range: 6 posterior to 22 anterior ), 0 degrees abducted ( SD: 4.9, range: 10 adduction to 9 abduction ), and 2 degrees externally rotated (SD: 7.5, range: 17 internal to 13 external ). 
Mean movement of the pelvis from the initial lateral decubitus position to the dislocated position was 12 degrees posterior ( SD: 7.6, range: 25 posterior to 1 anterior ), 2 degrees abducted ( SD: 4.4, range: 6 adduction to 11 abduction ), and 7 degrees internally rotated (SD: 5.0, range: 15 internal to 1 external ).

Mean movement of the pelvis from the supine position to the dislocated position was 8 degrees posterior (SD: 8.6, range: 26 posterior to 6 anterior), 3 degree abducted (SD: 6.0 , range: 6 adduction to 20 abduction), and 4 degrees internally rotated (SD: 6.8 , range: 24 internal to 5 external ).

\section{Discussion}

One important factor affecting alignment of the acetabular component has been reported to be the orientation of the pelvis on the operating table ${ }^{2), 5), 8)}$. It is hypothesized that anterior tilting or internal rotation of the pelvis intraoperatively in the lateral decubitus position may lead to decreased anteversion of the acetabular component, as compared to the surgeon's intended orientation of the component. It is also hypothesized that adduction of the pelvis in the lateral decubitus position on the operating table may lead to increased abduction of the acetabular component. However, in spite of the impact of these factors on clinical outcomes, there are few reports on measuring pelvic tilting angle, abduction angle, and axial rotation angle in the lateral decubitus position on the operating table.

This study showed that the pelvis was not always placed in neutral, despite the surgeon's intention. It was further tilted posteriorly and rotated internally when the socket was inserted. Movement of the pelvis from the initial lateral decubitus position to the dislocated position was larger than that of the pelvis from the supine position to the initial lateral decubitus position.

With conventional mechanical alignment guide systems, mean 8 degrees posterior tilting of the pelvis from the supine position to the dislocated lateral decubitus position may lead to 8 degrees less anteversion of the acetabular component. Mean 3 degrees abduction of the pelvis seemed to have a small effect on the cup abduction angle, but variation of the pelvic abduction angle on the table is not insignificant. Mean 4 degrees internal rotation of the pelvis may lead to an additional decrease in the cup anteversion with conventional mechanical alignment guide systems. This study demonstrated that mean 12 degrees of anteversion may be overestimated, and the cup may be placed in less anteversion than intended, when using mechanical guide systems. The variation of the pelvic tilt on the operating table also seemed to be an issue with regard to socket malposition. Therefore, intraoperative threedimensional measurement of pelvic orientation is necessary to place the socket without a large bias and variation.

\section{References}

1. Abel, M.F., Sutherland, D.H. : Evaluation of CT scans and 3-D reformatted images for quantitative assessment of the hip. J. Pediatr. Orthop. ( 1994 ); $14: 48-53$

2. DiGioia, A.M., Jaramaz, B., et al : Image guided navigation system to measure intraoperatively acetabular implant alignment. Clin. Orthop. ( 1998 ); 355: 8-22 
3. D'lima, D.D., Urquhart, A.G., et al : The effect of the orientation of the acetabular and femoral components on the range of motion of the hip at different head-neck ratios. J. Bone and Joint Surg. ( 2000 ); 82-A(3): 315-321

4. Kennedy, J.G., Rogers, W.B. : Effect of acetabular component orientation on recurrent dislocation, pelvic osteolysis, polyethylene wear, and component migration. J. Arthroplasty (1998); 13: 530-534

5. McCollum, D.E., Gray, W.J. : Dislocation after total hip arthroplasty : Causes and prevention. Clin. Orthop. (1990) ; 261: 159-170

6. Murray, D.W. : The definition and measurement of acetabular orientation. J. Bone Joint Surg. (1992) ; 75B : 228-232

7. Robinson, R.P., Simonian, P.T., et al : Joint motion and surface contact area related to component position in total hip arthroplasty. J. Bone and Joint Surg. (1997 ) 79-B(1): 140-14

8. Woo, R.Y.G., Morrey, B.F. : Dislocations after total hip arthroplasty. J. Bone Joint Surg. (1982) ; 64A: 1295-1306 